\title{
ENFOQUES Y DEBATES SOBRE RECURSOS NATURALES, ACUMULACIÓN Y TERRITORIO*
}

\author{
Recibido: 23 de agosto de 2018 • Aprobado: 11 de marzo de 2019 \\ https://doi.org/10.22395/seec.v22n51a6
}

\section{Silvia Gorenstein ${ }^{* *}$}

\section{RESUMEN}

El trabajo aborda enfoques del debate teórico actual en torno a las actividades basadas en la explotación de recursos naturales (RRNN) y su rol en el proceso de acumulación territorial del presente ciclo de capitalismo. En esta revisión se contemplan abordajes de la economía política y del campo de las ciencias sociales, identificando sus derivaciones teóricas en la caracterización de los RRNN y las actividades extractivas, la cuestión de la renta en la integración a cadenas globales de valor, el rol del Estado y, más en general, las repercusiones para los procesos de desarrollo de países de Latinoamérica, en los que se observan crecientes disputas territoriales por el uso intensivo de los mismos.

\section{PALABRAS CLAVE}

Renta; cadenas globales de valor; disputas territoriales.

\section{CLASIFICACIÓN JEL}

N50, O13.

\section{CONTENIDO}

Introducción; 1. Primeras consideraciones analíticas; 2. Desde la "maldición" a las nuevas "oportunidades" de los recursos naturales; 3 . La renta en épocas de cadenas globales de valor y lógicas financieras; 4. Recursos naturales y acumulación: territorios en disputa; 5 . Conclusiones; Bibliografía.

Este trabajo se ha realizado en el marco del Proyecto Recursos naturales y nuevas territorialidades en la Argentina contemporánea (PICT 0312, Agencia Nacional de Promoción Científica y Tecnológica).

** Economista, Investigadora del Consejo Nacional de Investigaciones Científicas y Tecnológicas (Conicet). Especialista en Planificación Regional, Instituto Latinoamericano de Planificación Económica, Cepal. Posgrado en Desarrollo Económico y Planificación, Instituto Latinoamericano de Planificación Económica y Social, Cepal Santiago de Chile, Chile. Magíster en Economía, Universidad Nacional del Sur, Buenos Aires, Argentina. Miembro del Consejo Científico de la Red Iberoamericana de Investigadores en Globalización y Territorio (RII); Investigadora en el Centro de Estudios Urbanos y Regionales (CEUR-Conicet), Buenos Aires, Argentina. Correo electrónico: silvia.gorenstein@gmail.com. 


\title{
APPROACHES AND DEBATES ABOUT NATURAL RESOURCES, ACCUMULATION AND TERRITORY
}

\begin{abstract}
The paper addresses approaches to the current theoretical debate about activities based on the exploitation of natural resources (RRNN) and their role in the territorial accumulation process of the current cycle of capitalism. This review contemplates approaches to political economy and social sciences fields. It identifies their theoretical derivations in the characterization of RRNNs and extractive activities, income in the integration of global value chains, the role of the State and more generally, repercussions for development processes in Latin American countries, in which there are growing territorial disputes over their intensive use.
\end{abstract}

\section{KEYWORDS}

Rent; global value chains; territorial disputes

\section{JEL CLASSIFICATION}

$\mathrm{N} 50, \mathrm{O} 13$.

\section{CONTENT}

Introduction; 1. First analytical considerations; 2. From the "curse" to natural resources new "opportunities"; 3 . Income in times of global value chains and financial logics; 4. Natural resources and accumulation: disputed territories; 5. Conclusions; Bibliography.

\section{ABORDAGENS E DEBATES SOBRE RECURSOS NATURAIS, ACUMULAÇÃO E TERRITÓRIO}

\section{RESUMO}

Este trabalho apresenta abordagens do debate teórico atual sobre as atividades baseadas na exploração de recursos naturais (RN) e seu papel no processo de acumulação territorial do presente ciclo do capitalismo. Nesta revisão, são trazidas abordagens da economia política e do campo das Ciências Sociais, identificando suas derivações teóricas na caracterização dos RN e das atividades extrativas, a questão da renda na integração a cadeias globais de valor, o papel do Estado e, mais em geral, as repercussões para os processos de desenvolvimento de países da América Latina, nos quais são observadas crescentes disputas territoriais pelo uso intensivo deles.

\section{PALAVRAS-CHAVE}

Renda; cadeias globais de valor; disputas territoriais.

\section{CLASSIFICAÇÃO JEL} $\mathrm{N} 50, \mathrm{O} 13$.

\section{CONTEÚDO}

Introdução; 1. Primeiras considerações analíticas; 2. Desde a "maldição" às novas "oportunidades" dos recursos naturais; 3 . A renda em tempos de cadeias globais de valor e lógicas financeiras; 4. Recursos naturais e acumulação: territórios em disputa; 5. Conclusões; Bibliografia. 


\section{INTRODUCCIÓN}

La discusión teórica sobre las actividades intensivas en recursos naturales (RRNN) en el proceso de acumulación y desarrollo exhibe cambios significativos desde el último cuarto del siglo XX. El marco de estos debates son las expresiones del presente ciclo del capitalismo global y financiarizado, los nuevos paradigmas tecnológicos, y su traducción en la división internacional del trabajo. Se producen y reproducen nuevas/viejas asimetrías modificando, a su vez, las jerarquías de los capitales a escala global y el escenario competitivo internacional con el liderazgo de nuevos jugadores en el comercio mundial. Tal es el caso de China, cuya irrupción como demandante e inversor ha tenido una repercusión significativa, particularmente, en los países latinoamericanos productores de materias primas ${ }^{1}$.

Las problematizaciones efectuadas desde la economía política, sumado a las diversas contribuciones del amplio campo de las ciencias sociales, tienen derivaciones teóricas tanto en la caracterización de los RRNN según ciclos de vida (renovables y no renovables) como en la consideración tradicional de las actividades extractivas y sus repercusiones territoriales.

En América Latina se ha generado una trayectoria analítica que tensiona los planteamientos seminales del estructuralismo latinoamericano, a su vez, se produce una progresiva atención y jerarquización de las problemáticas vinculadas a los riesgos ecológicos y medioambientales. En tal sentido, se observa un tratamiento creciente de los conflictos en torno a su utilización (agua, tierra, paisaje, bosques), se identifican las relaciones distributivas antagónicas y el choque de intereses, así como las connotaciones culturales, históricas, sociales, políticas y económicas que se traducen en disputas territoriales de diverso tipo y alcance.

En este artículo se analizan puntos de esta discusión para una mejor comprensión del resurgimiento que envuelve a la problematización en torno a las actividades intensivas en RRNN, asociadas a la especialización que caracteriza -con diferentes intensidades-a los países de Latinoamérica. Se realiza una exploración bibliográfica que, sin pretender ser exhaustiva en términos de autores y corrientes teóricas, tiene el objetivo central de identificar las principales continuidades y rupturas en el abordaje de esta temática.

La primera sección se centra en las tensiones que han surgido en la disciplina económica en el tratamiento de estos recursos a partir de la endogenización y valorización de la naturaleza y sus atributos específicos. Las dos secciones siguientes

Con su estrategia de industrialización, China se integra al circuito capitalista y a la economía mundial impulsando una fracción importante de la demanda de materias primas y alimentos. 
presentan contribuciones que conectan a los RRNN con los debates sobre los procesos de acumulación y desarrollo desde la perspectiva general y latinoamericana. Sobre esta base, en la cuarta sección se introduce la cuestión de la renta, su apropiación y distribución, frente a los límites impuestos por las dinámicas de integración a cadenas globales de valor (CGV) y los que devienen del accionar del Estado. En las reflexiones finales se estilizan los puntos del debate teórico que, desde una perspectiva general, remiten a nuevas críticas e interrogantes sobre las posibilidades de acumulación y desarrollo basado en actividades intensivas en recursos naturales.

Entre otras cuestiones, esta discusión contribuye a la visibilización del proceso de degradación ambiental que se asiste a nivel global desde mediados del siglo XX, propiciando ciertas formas de control en los esquemas de producción basados en RRNN. Por su parte, las cuestiones controversiales en torno a la gobernanza de los mismos, particularmente en los países periféricos, advierte sobre las limitaciones que suponen los procesos de apropiación de rentas en el marco de las potenciales o efectivas integraciones en cadenas globales de valor bajo las transformaciones y nuevas dinámicas del capitalismo.

\section{PRIMERAS CONSIDERACIONES ANALÍTICAS ${ }^{2}$}

Los RRNN tienen múltiples definiciones que difieren según los fines de estudio o la óptica de la disciplina específica con que se abordan. Desde una perspectiva general, se definen en función de la capacidad de la naturaleza para satisfacer necesidades humanas utilizando directamente los elementos que forman parte del sistema natural o por transformación de algunos de ellos en materiales que, a su vez, pueden ser usados como materias primas para la producción de otros bienes. Entre estos se encuentran los elementos vitales, no apropiables ni intercambiables en el mercado como el aire, la energía solar y, hasta hace poco, el agua 3 .

La economía de los recursos naturales, que se ocupaba del estudio de la asignación de recursos escasos (reservas pesqueras, plantaciones, agua dulce, hidrocarburos, etc.), desarrolló esquemas formalizados con la idea de que cualquier recurso natural tiene una tasa óptima de uso (o de explotación en el caso de recursos agotables). El primer cambio en este enfoque, se produce a partir de la segunda

2 Esta sección ha contado con aportes de Mónica Castro, becaria doctoral PICT 2017, Agencia Nacional de Promoción Científica y Tecnológica.

3 El agua y el aire son los ejemplos típicos de bienes libres mencionados en la teoría económica convencional por considerarse abundantes y, por lo tanto, con un valor marginal nulo para cualquier usuario real o potencial. Se diferencian de los llamados bienes públicos, en general provistos por el Estado -alumbrado de calles; seguridad; parques, entre otros-, cuyo consumo es indivisible porque puede ser consumido por todos los miembros de una comunidad. 
posguerra cuando comienzan a visibilizarse ciertos efectos relacionados con las repercusiones ambientales de la actividad productiva. La economía ambiental (EA), enrolada en la teoría económica neoclásica, pondrá el acento en las externalidades y la asignación óptima de los recursos agotables, así como en las mediciones de los efectos contaminantes de los procesos productivos. El concepto de externalidad aplicado a los RRNN, siguiendo a Aguilera y Alcántara (1994) y, más recientemente, a Burriel (2012), se asocia a una falla de mercado y, en algunos casos (aire, río, otros bienes libres), a la ausencia o indefinición de los derechos de propiedad ${ }^{4}$.

El andamiaje teórico de la llamada economía ecológica (EE) -desarrollado desde mediados de los años 80 - produce un giro sustantivo en los debates sobre la temática. Las críticas a la EA reflejan, particularmente, la visión de la economía como un sistema abierto, en íntima y recíproca relación con otros sistemas. De acuerdo con Martínez Alier (1995), el reduccionismo económico de la EA limita las respuestas a los interrogantes respecto de cómo traducir daños físicos futuros e inciertos a precios actuales. El concepto de externalidad revelaría, entonces, la incapacidad de dar valores a efectos futuros, inciertos o desconocidos. En tal sentido, Pengue (2009, p. 48) se pregunta: "Cómo aplicar estos conceptos cuando no se conocen los deseos o las preferencias de las generaciones futuras, cuando hay valores en conflicto, cuando se debe considerar el derecho a la existencia de las otras especies (o no), cuando hay enorme cantidad de grupos sociales relegados o cuando hay serios conflictos de valores".

El esquema analítico de la EE se asienta, a grandes rasgos, en el reconocimiento de la existencia de los límites de la tierra y el medio ambiente para soportar la actividad económica. En las restricciones al crecimiento se combinan la oferta finita de RRNN y la capacidad que tiene la naturaleza para absorber los deshechos e impactos ambientales (contaminantes sólidos, químicos tóxicos, emisiones de gases) ocasionados por el hombre. Los análisis desde esta perspectiva contemplan, entonces, cuestiones económicas y ambientales interiorizando, entre otros aspectos, riesgos relacionados con el cambio climático y los desastres naturales mientras que los abordajes sobre impactos medioambientales derivados de la producción y el consumo articulan perspectivas sociales, políticas y ecológicas.

En suma, desde la EE - de naturaleza transdisciplinaria- se aproximan conocimientos sobre las bases materiales que hacen que el funcionamiento de los sistemas productivos sea sostenible. Se evalúa el consumo de recursos, con la carga ambiental asociada, contabilizando los flujos de energía y materiales que se ponen en juego en todas las etapas de producción, circulación y consumo. En cambio, la llamada

Por ejemplo, el abuso en el consumo individual en situaciones de utilización de recursos que son compartidos por un número apreciable de individuos, como el agua de un río. 
EA -dentro del maestreen de la disciplina- sigue poniendo el foco en el sistema de precios como base para la asignación óptima de los recursos y de los sujetos de contaminación, ignorando o relegando analíticamente a los factores que degradan los ecosistemas y la distribución de los beneficios/costos ambientales.

Hay otras interpretaciones que nutren la actual discusión teórica en torno a los RRNN donde convergen diversos aportes que introducen nuevas categorías analíticas y, en particular, herramientas para identificar y cuantificar los costos del proceso productivo que generan repercusiones negativas sobre la naturaleza 5 . En esta dirección, interesa aquí señalar las siguientes:

- Las relaciones entre la naturaleza y la sociedad pueden asumir modalidades diversas dependiendo, básicamente, de las características del régimen de producción ${ }^{6}$. En el capitalismo, se definen modalidades de uso que facilitan los requerimientos de la acumulación y reproducción del capital utilizando en forma masiva los elementos naturales existentes, en forma directa o a través de complejos procesos de transformación que, entre otros, reflejan la disponibilidad de conocimientos científico-tecnológicos, la posibilidad de adquirirlos y la capacidad tecnoeconómica para utilizarlos. De este modo, y a partir de las formas de movilización que los actores sociales establecen con la técnica, la materia se transforma en recurso, evolucionando como producto cuyas propiedades pueden incrementarse en el tiempo, como también perder valor frente al descubrimiento de nuevas materias con propiedades que satisfacen necesidades. En tal sentido, Raffestin (2011, pp.158-160) reflexiona sobre la falsedad del atributo natural del recurso, dado que los mismos son manifestaciones de las relaciones políticas, sociales y económicas que la humanidad establece con la materia, asignando valor a sus propiedades en diferentes etapas históricas. Es decir, es el hombre quien, mediante su práctica, adjudica propiedades a la materia, convirtiéndola en valiosa a los fines de satisfacer sus necesidades, las cuales se van transformando en el transcurso del tiempo ${ }^{7}$.

Por ejemplo, los costos de regeneración o descontaminación de los recursos renovables o, entre otros, los que afectan la salud de la población.

6 Las sociedades primitivas utilizaban el ambiente natural por simple ocupación, mientras que en sistemas donde rigen relaciones de tipo campesino o en comunidades de pueblos originarios -aún los insertos en sociedades capitalistas- hay tendencias a la preservación y su uso con poca modificación (tala de bosques para obtener leña) o involucrando su transformación para obtener los recursos (desmonte para generar tierras de cultivo).

7 Solo a título de ejemplo el valor asignado a las propiedades del carbón como principal medio de combustión -que sustentó los procesos de industrialización de principios del siglo XVI de Europa-se modificó con el desarrollo de nuevas prácticas, ancladas en el conocimiento y la técnica, que le asignaron valor a otras materias energéticas. Un ejemplo más reciente, microorganismos como las algas, que solo constituían materia hasta hace unos pocos años, hoy son considerados una importante fuente para el desarrollo de biocombustibles de cuarta generación. 
- La clásica distinción económica entre RRNN renovables o no renovables estaría cada vez más devaluada. Todos ellos serían pasibles de ser agotados o contaminados por la utilización irracional a los que se los somete periódicamente, en el marco de las relaciones sociales que la definen. En este sentido, Seoane (2012) plantea que la capacidad de regeneración de los renovables se desarrolla a un ritmo más lento que el de su producción, alterando el carácter no renovable de los recursos afectados, puesto que la tasa de extracción supera a la de recuperación del recurso. Ello hace posible, entonces, que estos se extingan en gran parte, desdibujando la clásica distinción de los recursos naturales.

- Otras posturas teóricas sostienen la diferencia (renovables o no) y rebaten los pronósticos más pesimistas en torno a la agotabilidad de los RRNN. Por un lado, se argumenta que el proceso de agotamiento físico es variable e ineludible, derivado de su agotamiento intrínseco e, incluso, de previos cambios significativos provocados por otras alternativas naturales o tecnológicas que deterioran o anulan su valor económico. Por otro lado, se enfatiza en ciertas fuerzas capaces de compensar sus limitaciones (agotabilidad), como el cambio tecnológico y la sustitución del recurso por factores de producción artificiales ${ }^{8}$. Sin embargo, se reconoce que las posibilidades tecnológicas capaces de revertir la agotabilidad del recurso suponen elevadas restricciones económico-financieras(Sánchez, 2005).En los hidrocarburos y los minerales, por ejemplo, si bien existen alternativas tecnológicas que podrían alterar su ciclo de vida, esta posibilidad está limitada por los elevados costos de la producción de escala derivados de factores económicos específicos (altos costos hundidos; mayor riesgo e incertidumbre inversora asociados a la geología, etc.). En tal sentido, y como en otras actividades, ello se refleja en el poder de mercado y las estrategias de inversión de los grandes jugadores globales que detentan la mayor porción de las reservas mundiales.

- Los desarrollos contemporáneos -en particular los de la economía ecológica- analizan una serie de procesos y fenómenos que hacen a la protección de los RRNN a través del cumplimiento de objetivos ambientales (biodiversidad, retención de carbono, etc.), alertando en torno a los límites biofísicos y la alteración en los ciclos biogeoquímicos derivados de los cambios profundos que se están produciendo en la apropiación de los recursos, el consumo y las distintas demandas. Al respecto Pengue (2015, p.73) señala que:

\footnotetext{
Un ejemplo en este sentido es la exploración-explotación de reservorios de hidrocarburos no convencionales a partir del uso de la sísmica 3D en la exploración, inversiones en la automatización de operaciones remotas, la perforación con alta presión a altas temperaturas, el fracking en varias etapas y la geonavegación a distintas profundidades y superficies.
} 
Los estudios vinculados a las tasas metabólicas de las sociedades ayudan a dar pistas sobre mejores formas de utilización de los recursos de base (suelo, tierras, minerales, metales, petróleo, biomasa, materiales) hoy enfrentados a una realidad: los claros límites físicos que tienen las sociedades no sólo en el acceso sino en la disponibilidad de tales recursos, si el mundo sigue solamente enfocado en su espiral de consumo y producción.

- La mercantilización de los RRNN alcanza, cada vez más, a los considerados libres o comunes. El caso del agua, así como los bonos de carbono, son una muestra clara del alcance que adquiere la valorización financiera en el actual ciclo del capitalismo globalizado. Por su parte, los avances científicos y tecnológicos profundizan campos de aplicación basados en RRNN, abriendo paso también a un profundo proceso de mercantilización de la naturaleza. Las biociencias son cada vez más utilizadas en los procesos productivos, y se incorporan nuevos conocimientos y procedimientos biotecnológicos al tratamiento de los recursos que alteran, entre otros, su estructura genética. La bioeconomía también gana terreno en términos teóricos, lo cual redefine patrones productivos tradicionales, buscando producir más con menos RRNN. Sus métodos de valoración generan nuevos indicadores, como la huella ecológica, huella hídrica, huella de carbono, agua virtual ${ }^{9}$ y la utilización de técnicas cualitativas para los análisis sociológicos. Estos indicadores aportan elementos tanto para el análisis de las relaciones comerciales que se establecen entre los países, en particular los especializados en exportaciones de productos primarios, como en términos de posicionamiento relativo en estos escenarios ${ }^{10}$.

En síntesis, la creciente importancia concedida a la ecología y los aspectos ambientales conducen a la endogenización de la naturaleza en las formas de asignar valor a los RRNN, aquí se encuentran, al igual que en otras problemáticas socioeconómicas, visiones teóricas diferentes. En estas discusiones hay, a su vez, nuevos y viejos abordajes que ponen el acento en el rol de las actividades intensivas en RRNN en los procesos de acumulación y desarrollo de países/regiones.

9 Sintéticamente: i) La huella ecológica, estima el peso en términos de requerimientos de consumo de recursos y asimilación de desechos de una determinada población o economía, expresados en áreas de tierras productivas; ii) la huella hídrica, por su parte, contempla el agua como recurso central para la reproducción social y se define como la medida entre el agua necesaria para producir cada uno de los bienes y servicios que son utilizados; iii) el concepto de agua virtual mide la cantidad de agua necesaria para producir bienes y servicios no siempre consumidos en los países en los que se originan (Wackernagel y Rees, 2001; Ganem y Peinado, 2012).

10 Por ejemplo, Argentina es uno de los mayores exportadores de agua virtual con 98 Gm³/año detrás de Estados Unidos, China, India y Brasil. La huella de agua promedio es de $1.607 \mathrm{~m}^{3} /$ anuales per cápita, frente a una huella hídrica mundial promedio de 1.385 m³/anuales per cápita. Esto coloca a Argentina como un país con una alta utilización de sus importantes recursos hídricos (Ganem y Peinado, 2012). 


\section{DE LA “MALDICIÓN" A LAS NUEVAS “OPORTUNIDADES" DE LOS RECURSOS NATURALES}

El interés por los recursos naturales está presente desde los orígenes de la teoría económica. Los mercantilistas sostenían que la riqueza de las naciones se asociaba a la posesión de oro y plata, mientras que los autores clásicos, incluyendo a los fisiócratas, la atribuían fundamentalmente al valor de la tierra, el agua y los productos agrícolas. En tal sentido, Piketty (2013, p. 61) advierte que:

Estas formas de riqueza de la sociedad deben incluirse en el capital, distinguiendo entre acumulación y apropiación, dado que es difícil separar al valor de las tierras "vírgenes" (como las descubiertas por el ser humano hace siglos o milenios) del de las múltiples mejoras -drenaje, irrigación, barbechos, etc.- aportados por el ser humano a tierras agrícolas. Los mismos problemas surgen con el petróleo, el gas, "tierras raras", en los cuales al definir su valor resulta complejo distinguir del de las inversiones que permitieron descubrir los yacimientos y explotarlos.

En la teoría clásica, los RRNN disponibles formaban parte, con otros factores tangibles e intangibles (mano de obra, equipamiento, cultura, etc.), de la capacidad productiva potencial de un sistema económico, mientras que el libre comercio y la división del trabajo internacional, con base en las ventajas comparativas, contribuía a movilizar y acrecentar los límites de dicha capacidad. Siguiendo esta tradición teórica, una parte significativa del pensamiento económico del siglo XX también sostenía que la posesión de abundantes recursos naturales (tierras agrícolas, minerales, hidrocarburos, etc.) generaba potenciales ventajas en términos de desarrollo económico.

El cuestionamiento a estas posibilidades, asociadas a una estructura productiva especializada en actividades basadas en recursos naturales, provienen de la corriente estructural de la Cepal, la teoría de la dependencia, la corriente del desequilibrio y otros aportes marxistas de la economía política (Emmanuel, 1972; Samir, 1978). En estas contribuciones, elaboradas en los años 60 y 70 del siglo anterior, dicha especialización era una de las claves para entender los problemas del desarrollo de países periféricos, en particular los latinoamericanos. Desde esta perspectiva, pusieron el acento en el intercambio desigual derivado de la exportación de commodities(agrícolas, mineros, energéticos) y el rol proveedor de materias primas hacia los países centrales; las relaciones de poder y dependencia bajo el binomio centro-periferia; la restricción externa con el esquema macroeconómico stop and go y, en términos más generales, la heterogeneidad estructural y los condicionantes para superar el subdesarrollo desde un esquema productivo primarizado y un sistema económico afectado cíclicamente por la restricción externa y el deterioro de los términos de intercambio. 
Por su parte, un autor de fuerte ascendencia en este campo teórico como Hirschman (1977), caracterizaba a las industrias extractivas (hidrocarburos, minería) como enclaves debido a su estructura intensiva en capital, la escasa posibilidad de impulsar encadenamientos y la propensión de las multinacionales a no invertir localmente y a repatriar sus beneficios. En tal sentido, destacaba la importancia de la vía fiscal y de la intermediación del Estado, reconociendo riesgos de desvío de rentas (rentseeking) ${ }^{11}$ o por la ineficacia de las políticas públicas de gasto e inversión.

La directa alusión a la maldición de los recursos naturales se difunde, durante las dos últimas décadas del siglo XX, a través de diversas contribuciones que contemplan tanto los factores de índole económica (menor crecimiento) como aquellos de carácter institucional y político (Auty, 1993; Aguirre, 2017). En rasgos estilizados, observan:

- Los países con RRNN abundantes, sumados a una alta participación de estos en las exportaciones, exhiben menores tasas de crecimiento económico porque se bloquean posibilidades de diversificación y modernización de la estructura económica. Ello se ilustra a través de la llamada enfermedad de la economía holandesa, originada tras el descubrimiento de yacimientos de gas, el aumento de estas exportaciones y la apreciación del tipo de cambio que, combinados en dicho país, afectaron la competitividad de las exportaciones manufactureras, al conjunto de la industria y al empleo en este sector.

- La transmisión de efectos macroeconómicos adversos por la inestabilidad derivada de la intensa volatilidad de los precios en los mercados internacionales de los commodities (primarios, mineros, energéticos). Se producen fluctuaciones en el ingreso de divisas y el tipo de cambio, afectando a las actividades conectadas con el sector exportador y, en términos generales, ejerciendo efectos desestabilizadores en los presupuestos públicos.

- Implicaciones político-institucionales derivadas del mal uso, despilfarro o malversación de los ingresos públicos generados por el recurso extractivo y la proliferación de mecanismos de corrupción y acaparamientos de rentas (rentseeking).

\footnotetext{
Anne Krueger (1974) lo asocia a las derivaciones del poder monopólico, señala que los monopolistas gastan recursos importantes para mantener sus privilegios, ya sea a través de cabildeos de las élites políticas o la corrupción en el servicio civil para crear una legislación que impida que nuevos competidores entren en una industria y de ese modo mantener la posición de monopolio en un mercado. Cuando se habla de un país y su riqueza de recursos, el poder monopólico adquiere doble significado. Por un lado, se maximizan ganancias derivadas de su poder de mercado, y, al mismo tiempo, se obtienen por explotar un recurso estratégico nacional que pertenece a todos los ciudadanos de ese país (Velic, Cerovik y Maradin, 2018).
} 
La instancia teórica más amigable con el nuevo ciclo de primarización en el continente surge, paradójicamente, en la cuna del pensamiento estructuralista latinoamericano -la Cepal ${ }^{12}$ - bajo la convergencia de los enfoques neoestructuralista ${ }^{13} \mathrm{y}$ neoschumpeteriano. La clásica penalización analítica en torno a la especialización

regresiva, basada en ventajas comparativas naturales, se revierte con argumentos favorables a las actividades primarias y su utilización como puente para una inserción internacional competitiva basada en capacidades tecnológicas y de innovación.

La división entre producción primaria y manufacturera es reemplazada por la de bienes de alta o baja calidad, definidos según el tipo y dinamismo de la actividad de innovación. En tal sentido, la materialización de la estrategia de acumulación debería articular un conjunto integrado por tres aspectos (Pérez, 2010; Lewkowicz, 2011):

- Las ventajas en las industrias de procesos alrededor de los recursos naturales con tecnologías para convertirse en proveedores de energía, materiales e insumos (básicos y especiales, naturales y sintéticos, macro y nano) y de productos biológicos (tradicionales y de avanzada, ecológicos y biotecnológicos).

- Alianzas locales e internacionales en las industrias de base minera, energética y biológica y las transformadoras agroindustriales, químicas y metalúrgicas, en las cuales hay espacios para mejorar e innovar con el nuevo paradigma.

- Una orientación de IED basada en las ciencias de la vida y de materiales para reunir condiciones que permitan dar el salto dentro de unas décadas, en el período de instalación de la próxima oleada tecnológica (alguna combinación de biotecnología, nanotecnología y materiales a pedido).

En esta misma línea, Marín (2016, p. 249) subraya que los avances en biología molecular y sintética, relativos a nuevos procesos para la minería (biolixiviación y biorehabilitación en cobre y oro) y la agricultura (semillas de soja transgénica y otras) en los casos de Argentina y Chile "ilustran el planteamiento respecto a la existencia de importantes y nuevas oportunidades para el conocimiento pionero y las soluciones innovadoras y nuevas, desarrolladas por los proveedores locales lo cual les permite insertarse en las cadenas globales de valor ocupando nuevos espacios".

12 Ver Ramos (1998).

13 El (neo)estructuralismo, combina un conjunto heterogéneo de enfoques del campo de la economía política (desde el poskeynesiano, pasando por el marxismo, los regulacionistas y evolucionistas). Pérez Caldentey (2015, p. 38) señala que existen siete áreas temáticas de convergencia entre ellos: i) lo metodológico, ii) la caracterización del sistema de relaciones económicas internacionales y los temas asociados a esta, incluido el origen y la propagación de los ciclos, la temática del crecimiento de largo plazo y la restricción externa, iii) la relación entre distribución del ingreso, acumulación y crecimiento y desarrollo, iv) la volatilidad e inestabilidad, v) el progreso técnico y la innovación, vi) la relación entre el corto y el largo plazo y vii) el papel del Estado/ gobierno. 
En consecuencia, para estas contribuciones, los sectores remolque del crecimiento pueden tener base en los recursos naturales de cada país en los cuales se desarrollarían y aplicarían tecnologías competitivas para insertarse en las cadenas globales de valor (CGV). Esta integración implicaría la coordinación y control de la difusión y cumplimiento de estándares productivos y tecnológicos, de modo que se garantice la eficiencia global de la cadena (competitividad) y las oportunidades de upgrading(escalamiento) de productores o empresas de países en desarrollo. De este modo, las nuevas oportunidades, asociadas a las especificidades y soluciones innovadoras locales, también impulsarían el desarrollo de proveedores locales.

El llamado súper ciclo de los commodities, resultado de los altos precios internacionales en los años $2000^{14}$, también motivó reflexiones contrarias a la inexorabilidad de la "maldición", aun con el reconocimiento de la volatilidad de los precios típica de estos mercados. El cambio de escenario estaría dado por el sostenimiento de una tendencia ascendente derivada de la incorporación de China como demandante. En otros términos, el crecimiento económico estaría ahora más estrechamente ligado al alza del precio de los productos básicos de lo que lo estaba en el siglo XX por la magnitud y las características de la población incorporada al consumo mundial de los mismos (López, 2010). Así, el crecimiento de las exportaciones de América Latina, sustentado en China desde el año 2000, pasó de 0,7 \% en 1990 al 9 \% en 2011, tendencia que se mantiene. La región se convirtió en su principal proveedor en soja, mineral de hierro, cobre, níquel, harina de pescado y otros productos primarios ${ }^{15}$.

En suma, en el capitalismo periférico latinoamericano, con abundantes recursos naturales, la llamada "maldición" podría convertirse en nuevas oportunidades transformando las ventajas comparativas naturales en ventajas competitivas, por la vía del dinamismo tecnológico y el aprovechamiento de mercados segmentados (alimentos, energía, etc.), sumado a la construcción de una adecuada gobernanza. La buena gobernanza de los recursos naturales en su esencia contempla e integra aspectos económicos, sociales, ambientales e institucionales en el marco

14 Hasta la intensificación de la crisis financiera durante la segunda mitad del 2008, las cotizaciones de los commodities subieron a ritmo firme. Así, entre el 2002 y 2008 los precios nominales de los alimentos y de los metales aumentaron del 170 \% y $290 \%$, respectivamente. Sin embargo, los valores récord de junio-julio de 2008 se recortaron como mínimo un 45 \% hacia fines de 2008 y comienzos de 2009, instalando una tendencia descendente (si bien variable entre materias primas). El precio del petróleo crudo, por ejemplo, cayó desde el pico de USD 145 por barril en julio de 2008 hasta llegar a 30,28 USD por barril en diciembre del mismo año e inicia una recuperación relativa en torno a los 100 USD por barril en el 2009.

15 En este escenario, Argentina y Brasil concentraron entre el 70 \% y 80\% en las exportaciones del complejo oleaginoso; Perú y Chile en cobre, hierro y sus derivados; el 87 \% de las venezolanas fueron en petróleo y sus derivados; las de Bolivia concentraron el $88 \%$ en minerales y sus derivados 
del desarrollo sostenible. En tal sentido, el rol del Estado, y la buena o mala calidad de la gestión pública, se consideran determinantes para obtener buenos resultados en términos de ingresos y bienestar del conjunto social involucrado (Altomonte y Sánchez, 2016).

Dos temas se destacan para las políticas públicas: i) el desarrollo de mecanismos virtuosos en el sentido de asegurar una inversión eficiente de las rentas extraordinarias de recursos naturales en época de precios altos. En particular, la creación de fondos contracíclicos para contrarrestar la futura volatilidad de precios ${ }^{16}$, o impulsar inversiones públicas en educación, salud, infraestructura e innovación y desarrollo tecnológico que a la postre puedan reemplazar los activos extraídos; ii) el mejoramiento de la gestión pública y la generación o fortalecimiento de capacidades institucionales para prevenir y resolver los conflictos socioambientales que surgen en el proceso de desarrollo de los sectores extractivos.

Estas ideas requieren, sin embargo, ciertas prevenciones críticas a la hora de vislumbrar potencialidades o bloqueos a los procesos de acumulación y desarrollo socioeconómico ligados a los RRNN.

\section{LA RENTA EN ÉPOCAS DE CADENAS GLOBALES DE VALOR Y LÓGICAS FINANCIERAS}

La explotación de RRNN implica la generación de una renta asociada a la rentabilidad superior (por encima de una tasa normal de ganancia) que se obtiene por las condiciones de escasez y su abundancia relativa en algunos países/regiones. El planteamiento inicial lo realizó David Ricardo (2014/1817), advirtiendo el carácter diferencial de la renta de la tierra, a partir de la heterogeneidad y no reproductibilidad de ese medio de producción natural. De este modo, frente al desplazamiento de la frontera agrícola y la puesta en producción de tierras de menor calidad los propietarios de las más fértiles (o mejor ubicadas) percibirían, bajo esta concepción, una retribución diferencial por el mayor rendimiento de las mismas.

Desde las contribuciones de Marshall (1890/1948) y Schumpeter (1954), la noción de renta económica también se asoció a las condiciones de producción que trascienden a un recurso natural, conservando el atributo diferencial. En términos muy sintéticos, las rentas de innovación se generan a través de la creación de nuevos productos o la explotación de atributos no explorados del mismo o por el desarrollo de procesos alternativos de producción y nuevos mercados (Schumpeter, 1954). En esta dirección, el concepto de cuasirrenta, formulado por autores evolucionistas y neoschumpeterianos, alude a un tipo de ganancia extraordinaria derivada de la

16 Se trata de una alternativa de política pública que consiste en establecer fondos de estabilización para mitigar el impacto presupuestario de crisis comerciales transitorias provocadas por la caída de precios internacionales. 
innovación que se erosiona una vez que se difunde a lo largo del sistema económico. Con este enfoque, siguiendo a Sztulwark y Braude (2009), el concepto de renta se define en el marco de un funcionamiento económico donde el proceso de innovación asume un carácter estructural, no necesariamente transitorio, y este proceso de generación y apropiación de rentas de innovación es clave para la comprensión de una economía capitalista en la medida que se asocia a su reproducción sistémica.

Siguiendo ambas tradiciones teóricas, Nochteff (1995) articula la noción de renta diferencial ricardiana con la definición de cuasirrenta neoschumpeteriana integrando el efecto del cambio tecnológico. Las primeras se convierten en cuasirrentas en los casos en que la escasez de un recurso natural es transformada por la introducción de una innovación tecnológica ${ }^{17}$. Asimismo, se pueden obtener cuasi rentas de privilegio por la existencia de barreras institucionales (reservas de mercado, privilegios de financiamiento, subsidios) incluyendo las que se generan por la escasez de recursos naturales sostenida por instituciones restrictivas. Es decir, este tipo de cuasirrenta puede sostenerse tanto como duren la escasez de los recursos naturales o las instituciones restrictivas (regulaciones, excepciones impositivas, etc.).

La diferencia entre una u otra cuasi renta no es neutral en términos de las dinámicas que estructuran la conformación y reproducción del capital en los países dotados de recursos naturales, tampoco lo son los límites o condicionantes para el desarrollo de capacidades tecnológicas e innovativas y, en consecuencia, la apropiación de rentas de esta naturaleza. Procesos que no pueden estar ausentes a la hora de analizar potenciales o efectivas integraciones en las difundidas $\mathrm{CGV}^{18}$.

17 Entre las innovaciones se destacan aquellas dominadas por los proveedores (Pavitt, 1984), como en la minería y otras industrias extractivas donde los proveedores de maquinarias e insumos realizan las innovaciones, y las basadas en la ciencia (descubrimientos científicos y su posterior modernización tecnológica) vinculadas, por ejemplo, a la biotecnología y la química.

18 Este concepto ha sido ampliamente difundido en el terreno de los estudios de casos (internacionales, nacionales, regionales) y en esferas de la política pública promovidas desde los organismos multilaterales de crédito. 
Este concepto traduce desde una perspectiva metodológica mesoeconómica las dos tendencias contemporáneas en los mercados: globalización de la producción y del comercio (y de los patrones de consumo) y los procesos de desintegración vertical de las grandes empresas multinacionales (EMN) a través de la externalización de funciones, la separación de la propiedad y la integración del control, facilitados por la difusión de las TIC, y la conformación de redes empresariales que traspasan las fronteras nacionales, tal como lo asumen Gereffi (2001) y Gereffi, Humphrey y Sturgeon (2005). Tres cuestiones esenciales surgen de este enfoque:

- Las modalidades de gobernanza de las cadenas, esto es, las formas de coordinación de empresas y productores ubicados en diferentes etapas productivas y comerciales y en diferentes localizaciones, de modo de garantizar la eficiencia (competitividad) global de la cadena, lo que implica la coordinación y control de la difusión y cumplimiento de parámetros (estándares) productivos y tecnológicos.

- Las oportunidades de upgrading (escalamiento) de productores/empresas de países en desarrollo gracias a su integración en las CGV. De este modo, la acumulación de un país, a través de esta actividad queda relacionada con el "lugar" que le cabe en la red mundial.

- Las asimetrías -económicas, financieras, tecnológicas y de información- se vinculan con ciertos nodos específicos de comando de estas organizaciones productivas (CGV comandadas por oferentes o CGV dominadas por compradores). En estos casos, buena parte de la renta es direccionada hacia dichos nodos a través de diversos mecanismos operativos: control de canales comerciales, mecanismos de premios y castigos y creación de barreras a la entrada, derechos de propiedad, entre otros.

El tratamiento de las asimetrías y relaciones de poder en las cadenas productivas no es novedoso. Las evidencias en países de América Latina, analizadas desde la tradición teórico-metodológica de los complejos/subsistemas productivos (agroalimentarios, regionales, territoriales) así como a partir de las contribuciones críticas (Gutman y Lavarello, 2012; Fernández, 2017; Gorenstein y Gutman, 2016) recientes al enfoque de CGV, revelan que:

- La integración a una CGV no solo garantiza la eficiencia colectiva de procesos fragmentados, dispersos geográfica y sectorialmente sino que, principalmente, viabiliza el ejercicio de poder económico de las empresas núcleo de las mismas a través de la conformación de jerarquías asimétricas de empresas y productores y de procesos parciales y controlados de derrame tecnológico (upgrading o catchig-up). 
- Las relaciones desde arriba hacia abajo y desde abajo hacia arriba que se configuran en una CGV no quiebran las limitaciones estructurales derivadas de los actores económicos de los países en desarrollo y, por lo tanto, los obstáculos para alcanzar el control de las cadenas a través de procesos colectivos de cooperación evitando su funcionalidad para la habitual integración asimétrica y subordinada.

- La captura de rentas cuasi monopólicas, a partir de la constitución de lo que Fernández (2016) identifica como "ámbitos de apropiación corporativa" (pp. 31-33), tornan difusos los límites entre lo público y lo privado. En este proceso, asimilable a la mencionada renta de privilegio, inciden particularmente los marcos regulatorios en función de intereses empresariales (privatización, tercerización y normatización administrativa).

Estas cuestiones se profundizan cuando se asocian las dinámicas propias de la financiarización, ligadas a los RRNN y las actividades intensivas en los mismos, del actual ciclo del capitalismo. En efecto, la incidencia del capital financiero tiene una expresión directa en el mercado internacional de los commodities y en los productos derivados (basados en materias primas agrícolas, mineras e hidrocarburos) a disposición de los inversores a gran escala, tales como los fondos de cobertura, los fondos soberanos, los fondos de pensiones, los fondos universitarios y otros inversores institucionales (Oxfam, 2012; Gorenstein y Ortiz, 2018).En consecuencia, la composición del capital -financiarización mediante-, de las potenciales actividades o fases integradas a una CGV no es una cuestión menor tanto en términos del lugar ocupado en la misma como en relación al carácter y destino de la renta que se apropia.

En definitiva, puntos de discusión importantes a la hora de contrastar el señalado cambio en la consideración de las actividades intensivas en RRNN subrayando los incentivos para la innovación de procesos y productos, bajo las nuevas posibilidades abiertas por las TIC, la biotecnología y la inserción en ciertas CGV.

\section{RECURSOS NATURALES Y ACUMULACIÓN: TERRITORIOS EN DISPUTA}

Los RRNN se han convertido en un foco de atención significativo en otros campos de las ciencias sociales. Diversos abordajes teóricos contemporáneos vinculan la revalorización y mercantilización reciente de los RRNN con la continuidad del proceso de acumulación originaria. Este proceso, asociado al cercamiento de los comunes de la etapa preindustrial en Gran Bretaña, consistió en la desarticulación y privatización de los espacios con derecho de uso colectivo para producir bienes 
de consumo familiar, liberando tierras para su uso agropecuario y generando una gran masa de población que luego se incorporarían como fuerza laboral al proceso de industrialización ${ }^{19}$. En tal sentido, Marx en El capital (1973, p. 624) describe e interpreta su funcionalidad:

La depredación de los bienes de la iglesia, la enajenación fraudulenta de las tierras del dominio público, el saqueo de los terrenos comunales, la metamorfosis llevada a cabo por la usurpación y el terrorismo más inhumanos, de la propiedad feudal y del patrimonio del clan en la moderna propiedad privada: de ahí otros tantos métodos idílicos de la acumulación originaria. Con estos métodos se abrió paso a la agricultura capitalista, se incorporó el capital a la tierra y se crearon los contingentes de proletarios libres y privados de medios de vida que necesita la industria de las ciudades.

Desde este enfoque, autores contemporáneos amplían y complejizan el análisis. Así, la idea sobre la producción social del espacio (Lefebvre, 1974) involucra la distinción entre espacios apropiados y espacios dominados. Los primeros son utilizados por una comunidad para servir a sus necesidades, mientras que los espacios dominados son los que se someten al interés de la producción capitalista. En esta separación y contradicción, según este autor, se constituye la dimensión espacial de la acumulación originaria y, en ese marco entonces, se puede vincular la intensificación contemporánea de la lógica de mercantilización de los RRNN.

Por su parte, la noción de acumulación por desposesión de Harvey (2005), refleja la lógica de la transformación de la naturaleza en mercancía, con la depredación de los bienes ambientales globales (tierra, aire, agua), dando continuidad al proceso de acumulación originaria a través de los reacomodamientos geográficos del capital y fuerza de trabajo excedente frente a las crisis de acumulación. La privatización de la tierra, la expulsión de poblaciones rurales hacia las ciudades y la reconversión de derechos de propiedad social (comunal, colectiva, estatal) en propiedad privada son fenómenos mencionados por este autor al dar contenido a dicha noción en el marco del denominado spatialfix.

El carácter permanente de los procesos de expropiación, como condición de existencia del capital, también aparece elaborado en contribuciones de otros autores. El elemento fundamental a considerar para esta corriente es el llamado extractivismo contemporáneo, que refleja una de estas formas reiteradas de acumulación originaria, mientras que a los nuevos enclosures se le pueden asociar rasgos de los antiguos cercamientos (Grigera y Álvarez, 2013; Midnight Notes Collective, 2012):

19 El término cercamiento de los comunes refiere en inglés a los commons, espacios que, mediante el derecho consuetudinario, quedaban fuera de las posesiones privadas. Estos espacios se desarticularon en el siglo XVIII durante la denominada revolución agrícola. 
- La antigua supresión del control comunal con el desplazamiento creciente de las actividades de autoproducción de pueblos originarios o campesinado en la actualidad.

- La entrega de tierras comunales a los acreedores por deudas de la Corona equiparable a la expropiación de tierras por deudas inducidas o efectuadas por la intervención de organismos como el FMI, el Banco Europeo, etc.

- los contingentes de fuerza de trabajo rural desplazada con la actual internacionalización de la misma en el marco de una creciente movilidad geográfica para garantizar bajos salarios, mayor flexibilización y menor poder organizativo.

La definición del extractivismo pone el acento en los procesos y fenómenos propios de las formas de explotación de los RRNN en el siglo XXI, particularmente en países de Latinoamérica. En este sentido, alude a tres características presentes: i) gran volumen o alta intensidad, ii) escaso o nulo procesamiento y iii) predominio de la orientación exportadora. Con las actividades extractivas tradicionales (hidrocarburos y minería) se incluye a la agricultura de exportación, configurada bajo el agronegocio en gran escala (especialmente la soja), a las plantaciones forestales y pasteras, a la pesca y, contemplando las modificaciones en la relación entre la tasa de explotación y la tasa de renovación de los bienes naturales, también se considera el turismo internacional.

Desde esta perspectiva, el modelo de producción profundiza la dinámica económica típica del enclave de exportación, considerando las características tecnoproductivas de las actividades (capital intensivo) ${ }^{20}$ y la naturaleza de los actores involucrados -corporaciones trasnacionales-(Gudynas, 2013; Seoane, 2012; Svampa, 2013). Es decir, el extractivismo es un modelo de acumulación que involucra: un alto consumo de RRNN; escalas de producción mucho más elevadas que anteriormente en las mismas actividades (minería a socavón/minería a cielo abierto; agricultura familiar/agronegocio, entre las principales), una localización determinada que, al estar en manos de grandes corporaciones, generan escaso valor apropiable por las comunidades de estos territorios y pueden desatar conflictos por repercusiones medioambientales o desplazamientos socioproductivos. Si bien se reconoce que la definición de condiciones favorables a este modelo fue impulsada durante el neoliberalismo económico, vigente en las décadas finales del siglo anterior, su

20 Se trata de actividades con procesos que utilizan equipamientos-maquinarias con tecnologías ahorradoras de mano de obra, así como de aquellas que demandan significativas inversiones (financieras, reales) para su puesta en marcha y funcionamiento como, por ejemplo, la minería, la actividad petrolera, la industria petroquímica, entre otras. 
consolidación se habría producido, siguiendo a Giarraca y Teubal (2010) desde el inicio del siglo XXI'21.

Estas ideas sobre el extractivismo o apropiación/desposesión se expresan en la práctica de numerosas y diversas organizaciones socioambientales, así como en los conflictos que se vienen produciendo en ciertos territorios. En efecto, el posicionamiento sociopolítico de este discurso ${ }^{22}$ ha significado un paso importante para la resistencia de estos movimientos sociales, visibilizando efectos como los ambientales y los desplazamientos socioproductivos (pueblos originarios, agricultura familiar, comunidades locales) derivados de actividades como la soja transgénica, la minería a cielo abierto, el fracking, las pasteras, etc. En términos del esquema conceptual propuesto por Lefebvre (1974), y más allá de sus resultados concretos, estas luchas representan la búsqueda de un contra-espacio, pues desafían la tendencia del capitalismo contemporáneo hacia la mercantilización de la vida social y las representaciones del espacio que resultan hegemónicas (Oslender, 2010, pp. 97-100).

\section{CONCLUSIONES}

La explotación y uso de los RRNN está inmersa en un amplio y diverso debate teórico en un contexto general que profundiza las lógicas del capital ligadas a los mismos. Los abordajes transitan desde el recurso en tanto insumo o sostén de las actividades productivas a la endogenización de la naturaleza y, en ese marco, las problemáticas en las que intervienen medios de producción naturales y los límites para el accionar humano.

Otros planos de discusión refieren a la especialización en actividades intensivas en RRNN en términos de acumulación y desarrollo económico. Los argumentos en torno a las oportunidades que se abren bajo los nuevos paradigmas tecnológicos y el impulso de estrategias de vinculación en CGV, se contraponen a los marcados por la corriente crítica al modelo extractivista que si bien contribuye a visibilizar diversas problemáticas socioambientales están lejos de interponer una perspectiva que contribuya a una mejor comprensión del potencial de acumulación de otras alternativas.

La limitación congénita de las estructuras productivas nacionales primarizadas, con un perfil exportador basado en materias primas, que fueran marcadas por

\footnotetext{
21 Svampa (2013) refiere al consenso de los commodities de los gobiernos neodesarrollistas, mientras que Gudynas (2013) alude al neoextractivismo progresista.

22 En tal sentido, el campo teórico más reciente de la ecología política cuestiona la distribución desigual de los costos y beneficios ambientales, promoviendo el activismo político organizado.
} 
los clásicos autores del estructuralismo latinoamericano (Prebisch, 1963, entre otros), no están ausentes en los nuevos debates. Con ello, los aspectos que fundamentan la mencionada maldición de los RRNN directa o indirectamente están presentes en estudios recientes que ponen en evidencia los diferentes contextos sectoriales externos y trayectorias internas con desigual exposición y grados de vulnerabilidad socioeconómica (Daher, Moreno y Aninat, 2017; Piquet, Tavares y Pessoa, 2017).

Las posibilidades del poder público nacional, de la mano de la conceptualización de la gobernanza, también es objeto de lecturas críticas teniendo en cuenta la actual fase del capitalismo en la que se profundizan las lógicas del capital desvinculadas de los territorios donde operan, con estrecha articulación a las modalidades de valorización financiera. Un punto crucial de discusión es el ejercicio de las funciones del Estado y los límites a su capacidad regulatoria. En las últimas décadas, particularmente en los países latinoamericanos, los instrumentos normativos han incorporado diversas cláusulas favorables a las empresas multinacionales inversoras, trato similar a los inversores locales, la posibilidad de utilizar las condiciones fijadas por otros tratados que pudieran ser más favorables a sus intereses, protección de las inversiones previas: tribunales supranacionales para la defensa de los intereses de los inversores externos en relación con actos de gobierno vinculados con las inversiones (Gorenstein y Ortiz, 2016).En este marco, se profundizan las tensiones que transforman al Estado "en un actor capturado" (Fernández, 2017 p. 337) por los agentes dominantes o con mayor capacidad de presión sobre las áreas de formulación e implementación de las políticas públicas que inciden en la materia decisivamente.

Por último, la instalación de la noción de desarrollo sustentable, al menos en el plano discursivo, intenta destacar que se está trascendiendo la lógica estrictamente económica al introducir aspectos esenciales de la matriz ambiental. En ese sentido, existe cierto consenso respecto de la necesidad de disminuir la presión sobre el ambiente, definiendo niveles de eficiencia en el uso de los recursos y buscando una transición socioecológica hacia trayectorias de sustentabilidad que implicarían un cambio en la matriz productiva y de consumo. Ahora bien, ello introduce otro plano importante de confrontación y debate en torno a los RRNN y sus posibilidades/límites en términos de acumulación y desarrollo. Muchas de las condiciones requeridas para generar o recrear procesos productivos sustentables (uso de energías más limpias y renovables, por ejemplo), replantearían liderazgos en términos de capacidades de inversión y desarrollos tecnológicos, desatando o intensificando conflictos en diferentes escalas (global, nacional, local) y tensionando, aún más, el posicionamiento de los países periféricos bajo este nuevo paradigma mundial. 


\section{BIBLIOGRAFÍA}

Aguilera, Federico y Alcántara, Vicent. (1994). De la economía ambiental a la economía ecológica. Barcelona: Icaria y Fuhem, 408 p.

Aguirre, Rafael. (2017). Recursos naturales y desarrollo: los dilemas de una relación crítica. Madrid: Instituto Universitario de Desarrollo y Cooperación, 100 p.

Altomonte, Hugo y Sánchez, Ricardo. (2016). Hacia una nueva gobernanza de los recursos naturales en América Latina y el Caribe. Santiago de Chile: Naciones Unidas, 258 p.

Auty, Richard. (1993). Sustaining Development in Mineral Economies: The Resource Curse Thesis. Londres: Routledge, 284 p.

Burriel, Óscar. (2012). Evolución del pensamiento económico sobre los recursos naturales. En: Información Comercial Española, ICE: Revista de Economía, n.o 865, p. 79-90. https:// dialnet.unirioja.es/servlet/articulo?codigo $=3997997$

Daher, Antonio; Moreno, Daniel y Aninat, Matías. (2017). Efectos socioterritoriales en Chile del súper ciclo de los commodities y de su término. En: Cadernos Metrópole, vol. 19, n.o 38, p. 127-155. Doi: doi.org/10.1590/2236-9996.2017-3805

Emmanuel Arghiri. (1972). El intercambio desigual: ensayo sobre los antagonismos en las relaciones económicas internacionales. Buenos Aires: Siglo XXI, 397 p.

Fernández, Víctor Ramiro. (2016). Desde el laboratorio neodesarrollista a la resurgencia neoliberal. Una revisión creativa del "doble movimiento" polanyiano en América Latina. En: Revista Estado y Políticas Públicas, n.o 7, p. 21 47.

Fernández, Víctor Ramiro. (2017). La trilogía del erizo-zorro. Redes globales, trayectorias nacionales y dinámicas regionales desde la periferia. Santa Fe: Anthropos, 397 p.

Ganem, Javier y Peinado, Guillermo. (2012). Estructura productiva, comercio internacional y ambiente. Indicadores biofísicos de la economía argentina desde la perspectiva del intercambio ecológicamente desigual. En: Decimoséptimas Jornadas Investigaciones en la Facultad de Ciencias Económicas y Estadística. Universidad Nacional de Rosario.

Gereffi, Gary; Humphrey, John y Sturgeon, Timothy. (2005). The governance of global value chains. En: Review of International Political Economy, vol. 12, n.o 1, p. 78-104. Doi: doi. org/10.1080/09692290500049805

Gereffi, Gary. (2001). Las cadenas productivas como marco analítico para la globalización. En: Problemas del Desarrollo, vol. 32, n.o 125, p. 9-37. Doi: doi.org/10.22201/ iiec.20078951e.2001.125.7389

Giarraca, Norma y Teubal, Miguel. (Comps.). (2010). Del paro agrario a las elecciones del 2009: tramas, reflexiones y debates. Buenos Aires: Antropofagia, 410 p.

Gorenstein, Silvia y Ortiz, Ricardo. (2016). La tierra en disputa: agricultura, acumulación y territorio en la Argentina reciente. En: Revista Latinoamericana de Estudios Rurales, vol. 1, n.o 2, p. 1-26.

Gorenstein, Silvia y Ortiz, Ricardo. (2018). Natural resources and primary sector-dependent territories in Latin America. En: Area Development and Policy, vol. 3, n.o 1, p. 42-59.Doi: 
doi.org/10.1080/23792949.2018.1431555

Gorenstein, Silvia y Gutman, Graciela. (2016). Desarrollo y territorio: clusters tecnológicos en la periferia. En: Revista Política e Planejamento Regional, vol. 3, n.1, p. 1-18.

Grigera, Juan y Álvarez, Laura. (2013). Extractivismo y acumulación por desposesión: un análisis de las explicaciones sobre agronegocios, megaminería y territorio en la Argentina de la posconvertibilidad. En: Theomai, n.o 27-28, p. 80-97.

Gudynas, Eduardo. (2013). Extracciones, extractivismos y extrahecciones: un marco conceptual sobre la apropiación de recursos naturales. En: Observatorio del Desarrollo, n.o 18, p. 1-18.

Gutman, Graciela y Lavarello, Pablo. (2012). Building capabilities to catch up with the biotechnological paradigm. Evidence from Argentina, Brazil and Chile agro-food systems. En: International Journal of Learning and Intellectual Capital, vol. 9, n.o 4, p. 392-412. Doi: 10.1504/IJLIC.2012.049616

Harvey, David. (2005). El "nuevo" imperialismo: acumulación por desposesión. Buenos Aires: Clacso, $33 \mathrm{p}$.

Hirschman, Albert. (1977). La estrategia del desarrollo económico. Ciudad de México: Fondo de Cultura Económica, 211 p.

Krueger, Anne. (1974). The Political Economy of the Rent-Seeking Society. En: American Economic Review, vol. 64, n.o 3, p. 291-303.

Lefebvre, Henri. (1974). La production de l'espace. París: Anthropos, 485 p.

Lewkowicz, Javier. (2011). Reportaje a Jorge Katz, referente del estructuralismo latinoamericano: "Con la soja no alcanza". Página/12.

López, Ramón. (2010). Crisis económicas mundiales, escasez de recursos ambientales y concentración de la riqueza. En: Revista de la Cepal, n.o 102, p. 29-50.

Marín, Anabel. (2016). Los recursos naturales como plataforma para el desarrollo de nuevas tecnologías y actividades. En: La Innovación y la Tecnología: ¿̇una ruta hacia el desarrollo? Conferencia Internacional Nuevos Enfoques para el Desarrollo Productivo. San José, 12 p. https://www.fes-mexico.org/fileadmin/user_upload/Publicaciones/Anabel__Marin_9.pdf

Marx, Karl. (1973). El capital (Tomo I). Ciudad de México: Fondo de Cultura Económica, 1016 p.

Marshall, Alfred. (1948). Principios de economía. Madrid: Aguilar, 973 p. Principles of Economy (1890) Master and Fellows of St Jonh's College, Cambridge;

Martínez Alier, Joan. (1995). De la economía ecológica al ecologismo popular. Barcelona: Icaria, $366 \mathrm{p}$.

Midnight Notes Collective. (2012). Los nuevos cercamientos. En: Theomai, n. ${ }^{\circ} 26$, p. 1 15.

Nochteff, Hugo. (1995). Los senderos perdidos del desarrollo: élite económica y restricciones al desarrollo en Argentina. En: Scarfi, Jorge (Ed.). El desarrollo ausente: restricciones al desarrollo, neoconservadorismo y élite económica en la Argentina. Ensayos de Economía Política. Buenos Aires: Norma, p. 21-156.

Oslender, Ulrich. (2010). La búsqueda de un contra-espacio: chacia territorialidades alternativas o 
cooptación por el poder dominante? En: Geopolítica(s). Revista de Estudios sobre Espacio y Poder, vol. 1, n. ${ }^{\circ}$ 1, p. 95-114

Oxfam. (2012). Annual Repport 2012-2013. Oxford: Oxfam, 96 p.

Pavitt, Keith. (1984). Sectoral patterns of technical change: Towards a taxonomy and theory. En: Research Policy, vol. 13, n.o 6, p. 343-373. Master and Fellows of St Jonh's College, Cambridge. Doi: doi.org/10.1016/0048 - 7333(84)90018 0

Pengue, Walter. (2009). Fundamentos de economía ecológica. Buenos Aires: Kaicron, 370 p.

Pengue, Walter. (2015). Recursos naturales, metabolismo social y desarrollo. En: Voces en el Fénix, n.o 43, p. $72-79$.

Pérez, Carlota. (2010). Dinamismo tecnológico e inclusión social en América Latina: una estrategia de desarrollo productivo basada en los recursos naturales. En: Revista de la Cepal, n.o 100, p. 123-145.

Pérez Caldentey, Esteban. (2015). Una coyuntura propicia para reflexionar sobre los espacios para el debate y el diálogo entre el (neo)estructuralismo y las corrientes heterodoxas. En: Bárcena, Alicia y Prado, Antonio (Eds.). Neoestructuralismo y corrientes heterodoxas en América Latina y el Caribe a inicios del siglo XXI. Santiago de Chile: Cepal, p. 33-92.

Piketty, Thomas. (2013). El capital en el siglo XXI. Ciudad de México: Fondo de Cultura Económica, $663 \mathrm{p}$.

Piquet, Rosélia; Tavares, Érica y Pessôa, João Monteiro. (2017). Emprego no setor petrolífero: dinâmica econômica e trabalho no Norte Fluminense. En: Cadernos Metrópole, vol. 19, n.o 38, p. 201-224.

Prebisch, Raúl. (1963). Hacia una dinámica del desarrollo latinoamericano. Ciudad de México: Fondo de Cultura Económica, 205 p.

Ramos, Joseph. (1998). Una estrategia de desarrollo a partir de complejos productivos en torno a los recursos naturales. En: Revista de la Cepal, n.o 66, p. 105-126.

Raffestin, Claude. (2011). Por una geografía del poder. Michoacán: El Colegio de Michoacán, 190 p.

Ricardo, David. (1817; 2004). Principios de economía política y tributación. Ciudad de México: Fondo de Cultura Económica, 332 p. Ricardo, David (1817), On the Principles of Political Economy and Taxation (1 edición), London

Samir, Amin. (1978). El desarrollo desigual: ensayo sobre las formaciones sociales del capitalismo periférico. Barcelona: Fontanella, 397 p.

Sánchez, Fernando. (2005). Bases conceptuales para la elaboración de una nueva agenda sobre recursos naturales. Santiago de Chile: Naciones Unidas, 59 p.

Schumpeter, Joseph. (1954). Capitalism, Socialism and Democracy. Londres: Routledge, 460 p.

Seoane, José. (2012). Neoliberalismo y ofensiva extractivista: actualidad de la acumulación por despojo, desafíos de nuestra América. En: Theomai, n.o 26, p. 1-28.

Svampa, Maristella. (2013). "Consenso de los commodities" y lenguajes de valoración en América Latina. En: Nueva Sociedad, n.o 244, p. 30-46. 
Sztulwark, Sebastián y Braude, Hernán. (2009). La adopción de semillas transgénicas en Argentina: un análisis desde la perspectiva de la renta de innovación. En: Desarrollo Económico, vol. 50, n.o 198, p. 297-319. Doi: 10.2307/41219103

Velic, Ismar; Cerovik, Ljerka y Maradin, Diego. (2018). Monopoly Exploitation and Rent-Seeking as an Inevitability of Capital Concentration. En: Asian Economic and Financial Review, vol. 8, n.o 4, p. 552-564. Doi: 10.18488/journal.aefr.2018.84.552.564

Wackernagel, Mathis y Rees, William. (2001). Nuestra huella ecológica: reduciendo el impacto humano sobre la tierra. Santiago de Chile: LOM, 207 p. 